\title{
Multi Medical Image Secure Hiding Technique at Multi Levels using Rational Operations and Compression
}

\author{
G. Thippanna ${ }^{1}$ and Dr. T. Bhaskara Reddy ${ }^{2}$ \\ ${ }^{1}$ Research Scholar, Dept. of CS \& T, SKU, Anantapuram, INDIA \\ ${ }^{2}$ Associate Professor, Dept. of CS \& T, SKU, Anantapuram, INDIA \\ 'gt.pana2012@gmail.com, ${ }^{2}$ bhaskarreddy_sku@yahoo.co.in
}

\begin{abstract}
Now a day's information security is most wanted achievement, and its need in the communication. The intends of this entitle work is wished-for an image encryption and decryption. Image encryption is an effective approach for providing security and privacy protection for medical images. This feature is especially very useful for multi medical images where the images can be making as one. In this work applies rational operators in between two images at multi levels to make multi key image to improve security levels. This work mainly approaches two operations that are $\mathrm{OR}$ and XOR logical operations.
\end{abstract}

Key Words: two medical images, Image compression techniques, logical operations

\section{Introduction}

In the networks/internets we are easily transmit data/information from one place to another. Here data/information and network bandwidth are proportional things. If the bandwidth size is low than the process high quality of data transmission is delay. So that such typed communications media provides security otherwise many hackers are ready to attack, and hack data/information from the communications. For image encryption and decryption the popular concept is Visual Cryptography Scheme (VCS)[1]. It was established by Naor and Shamir in the year of 1994. In this entitle work the main aim of this VCS concept to make two different images as one. Visual cryptography is a popular solution for image encryption. Here we used different type's keys to encryption and decryption of data. This encrypted data/information is easily transmitting through the communication media, and also if the hackers are hack information, but they couldn't understand, the secret image is decrypted without additional computations and any knowledge of cryptography [4]. The simplest Visual Cryptography Scheme is given by the idea of A secret image consists of a collection of black and white pixels where each pixel is treated independently [5]. There are many algorithms to encrypt the image in another image, but a few of them have been in visual cryptography for medical image. In this paper, the different approach has been produced for the visual cryptography for medical images.

This entitles paper Multi Image Secure Hiding Technique using Rational Operations wished-for an image encryption and decryption process. The main aims of this paper to overcome the problem of computer networks transmit the data with secure less. Cryptography is the concept to encrypt the image, and sends source to destination in the form of encrypted format. This encrypted image/information in the form unreadable format. It is also provides the security from the hackers. Follows this technique applies to same size of two images to merge into one and encrypt the images with same individual image size. Here no loss of information. Because we apply the few mathematical rational operation calculations on two different images such maketh encrypted image and send at a time two images from source to destination. And also we retrieve the same information using the same reverse calculations. When additionally we apply any compression 
technique on encrypted image its make the image in the form of unreadable and indecipherable and also reduce size of encrypted image. The principle of image encryption [2] will explain the details of encryption processor. Actually there mainly three types of keys [2] are available to encrypt the image.

\section{Image Processing Applications and Compression Techniques}

\section{a. Image Processing Applications}

There are three main applications in digital image processing [3] concepts.

They are:

a. Important pictorial information for human perception.

b. Image processing for autonomous machine application.

c. Effective storage and secure transmission.

But this entitles work related to effective storage and secure transmission application.

Using of VCS we encrypt the image into unreadable format, here we use a secrete key, if the hackers hack the image they couldn't know the original image because of they don't know the secrete key.

\section{b. Compression Techniques.}

The Definition of compression is to reduce the size of original. Applicable this definition to our concepts i.e. image processing, here every image consists of pixels and information. Apply the compression technique to images its reduces information, and repeated pixels which have the main content. Here in this our concepts image compression [6] basically two ways,

1. Lossy Compression: - after applies compression technique, at the time retrieve the original image it will lose some information, so that this typed compression technique is called lossy compression technique.

Example: JPEG, WEBP.

2. Lossless Compression:- after applies compression technique, at the time retrieve the original image it nothing will lose any information, so that this typed compression technique

is called lossless compression technique.

Example: WEBP, TIFF, JPEG 2000.

\section{Proposed Work}

\subsection{Forward Technique}

We start on our discussion with an example. Suppose the original image and their size of $\mathrm{M} \times \mathrm{M}$ pixels. If we apply our proposed technique on the photo of a old man and a family (cameraman) with $256 \times 256$ pixels is shown in Figure 1(a,b). Its key image at multi level shown in Figure 1(c, d), at multi level is obtained both information regarding the photo of Old man and family. If we only use the photo of baboon and pepper (128 $\times$ 128 pixels indicates), in Figure 2, Baboon, Madhuri (e, f). Its key image at multi level shown in Figure 2(g, h), at multi level is obtained both information regarding the photo of baboon and madhuri. The visual effect key images contain both images information, no one can easily identified the both images, and it is more helpful to send at a time more than one image in the network, if the adversary also don't dare to hack information in communications because of he couldn't understands. If we apply the image compression technique on encrypted image it's helpful to reduce the size of encrypted images and is 
more convenient to sends in the communication more than one image with less bandwidth of network bandwidth with securely. Here no data is lost when we applies this techniques. In some applications of image encryption, such a high-frequency components lost can be acceptable. Make a key element image by applying the conditional "OR" operation. This key element image is easily helpful to proceed the next step. Using this key element image with raw image -1 and raw image -2 apply the conditional operations i.e., "XOR" each other. To reduce the size encoded image compressed the image using Huffman compression technique. By this we get less size of image i.e. size reduced image and it is also helps to easily transfer even though through the low bandwidth network also as easily and firstly.

Figure -1 (256 X 256)

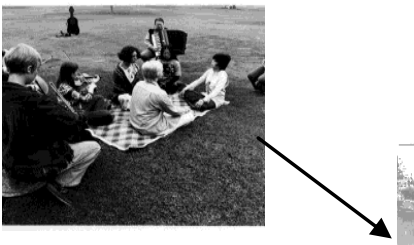

a

$1^{\text {st }}$ Level Key Image $\quad 2^{\text {nd }}$ Level Key Image

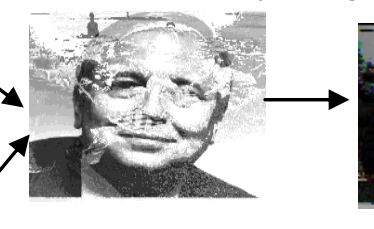

c

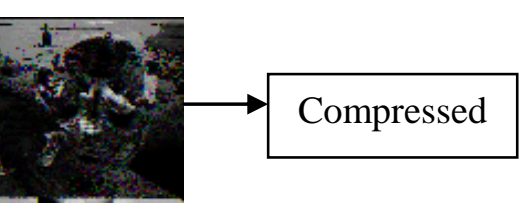

d

b

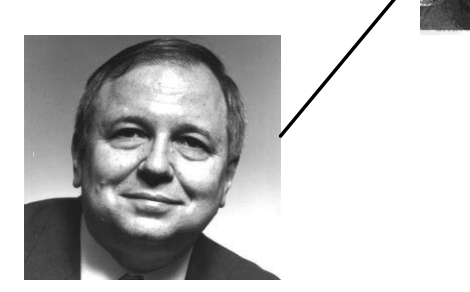

Figure -2

(128 X 128)

(1) $1^{\text {st }}$ level Key image $\quad 2^{\text {nd }}$ level Key Image

e
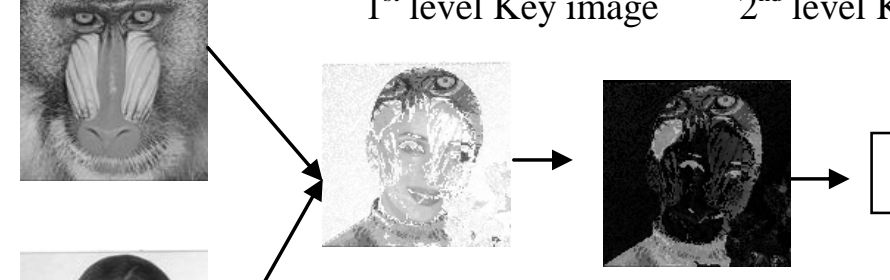

Compressed Image

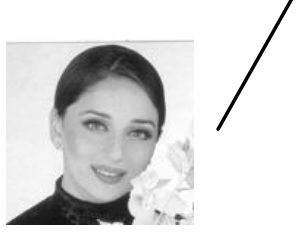

g

$\mathrm{h}$

f

OR Operation Between two two images

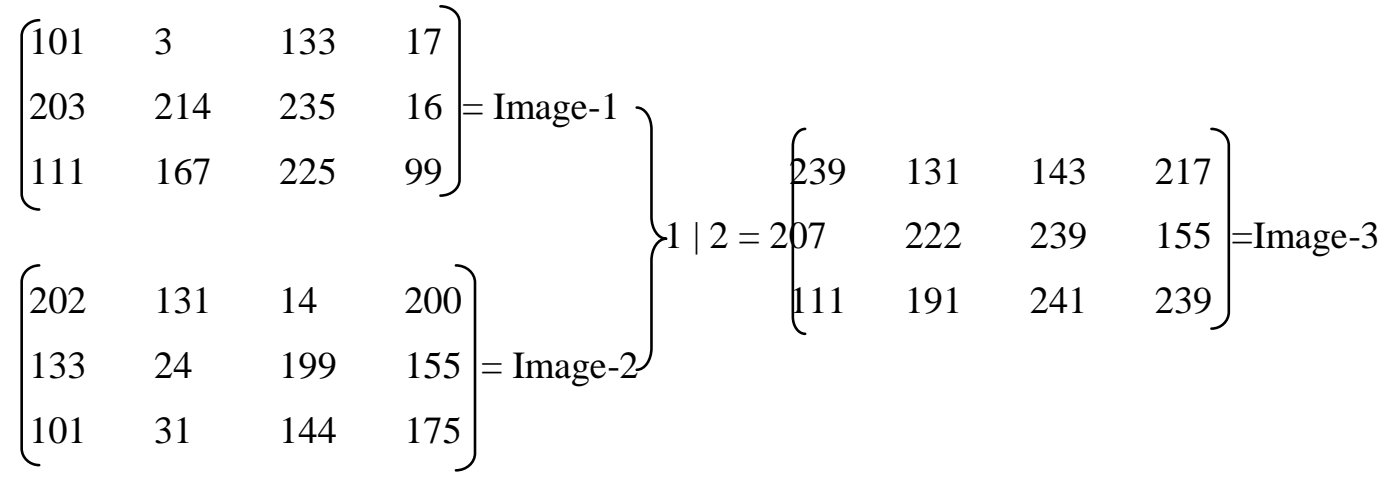


XOR Operation Between two Images

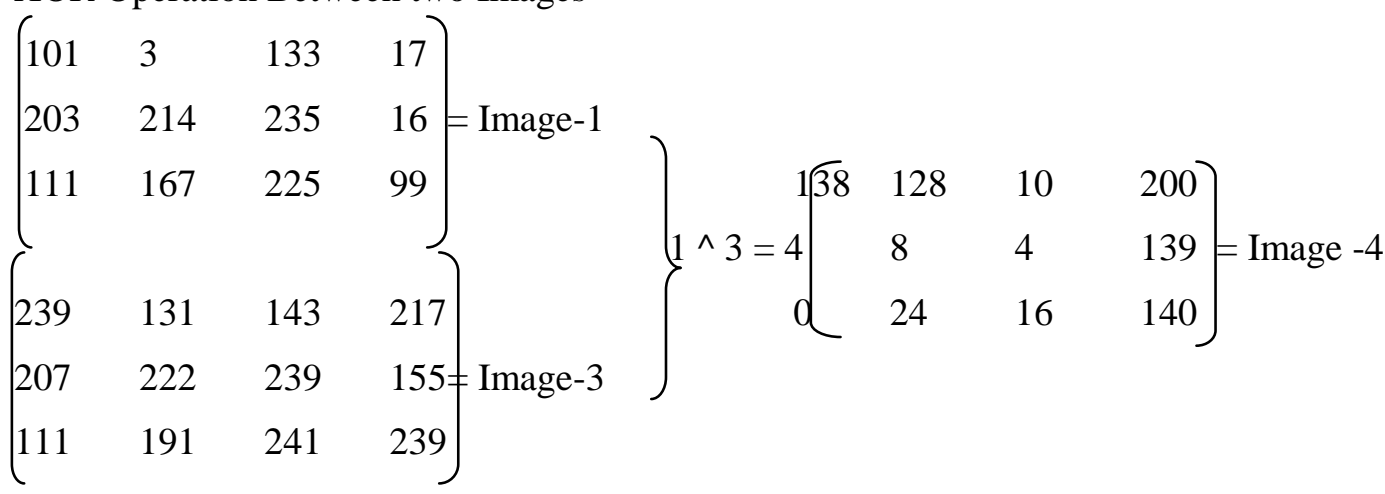

XOR Operation Between two Images

$\left.\left.\begin{array}{l}\left(\begin{array}{llll}202 & 131 & 14 & 200 \\ 133 & 24 & 199 & 155 \\ 101 & 31 & 144 & 175\end{array}\right]=\text { Image-2 } \\ \left(\begin{array}{llll}239 & 131 & 143 & 217 \\ 207 & 222 & 239 & 155 \\ 111 & 191 & 241 & 239\end{array}\right]=\text { Image-3 }\end{array}\right\} \wedge 3=\begin{array}{llll}37 & 0 & 129 & 17 \\ 4 & 198 & 40 & 0 \\ 10 & 160 & 97 & 64\end{array}\right]=$ Image -5

OR Operation Between two two images

$\left.\begin{array}{l}{\left[\begin{array}{llll}138 & 128 & 10 & 200 \\ 4 & 8 & 4 & 139=\text { Image-4 } \\ 0 & 24 & 16 & 140\end{array}\right]} \\ {\left[\begin{array}{llll}37 & 0 & 129 & 17 \\ 74 & 198 & 40 & 0= \\ 10 & 160 & 97 & 64\end{array}\right] \text { Image-5}}\end{array}\right\} 4 \begin{aligned} & \left.5=7 \begin{array}{llll}75 & 128 & 139 & 217 \\ 206 & 44 & 139 \\ 0 & 184 & 113 & 204\end{array}\right]=\text { Image }-6\end{aligned}$

The followed figure will explained in detail of proposed method. 


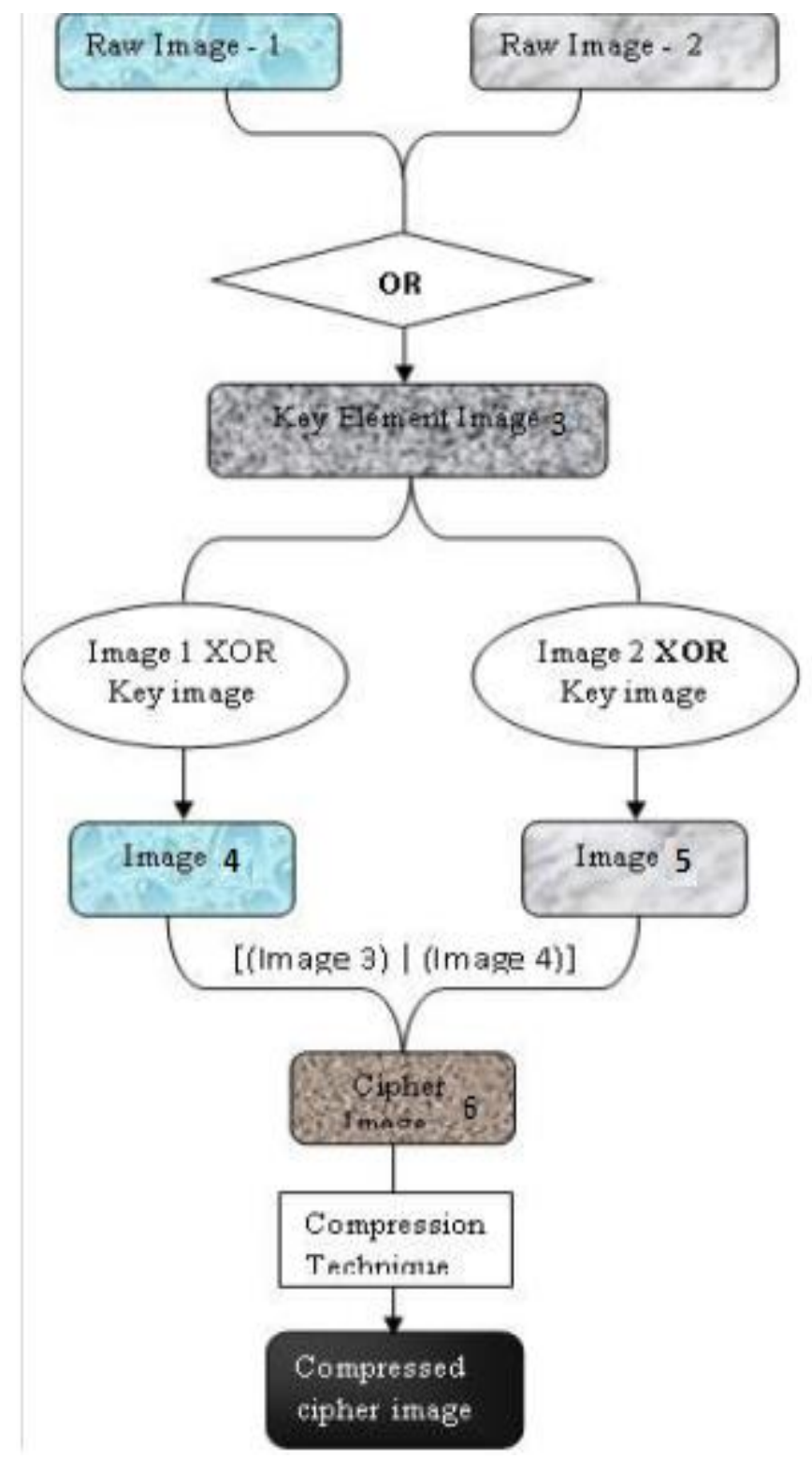

\section{Picture 1. Forward Technique for Proposed Method}

The followed figure will explained in detail of proposed method.

Step -1. Consider two same size raw images as an input files and read it.

Step - 2. Convert these images into digital format

Step - 3. apply (img-1.) | (img-2).

Step - 4. Found $[(\mathrm{img}-1) \mid(\mathrm{img}-2)]=\mathrm{img}$

Step - 5. Apply [(img -3) $\cup$ (img-1.] = img -4 file .

Step - 6. Apply $[(\mathrm{img}-3) \cup(\mathrm{img}-2)]=\mathrm{img}-5$ file

Step - 7. Apply [(img-4)|(img-5)] = img- 6 file. This is second level key image

Step - 8. Apply compression technique on step -7 images to generate compressed Image 


\subsection{Backward Technique}

We know already compressed cipher image is created; it should take as an input source in backward method. Uncompress the compressed cipher image, it delivers cipher image. Using the key element image apply XOR with cipher image it makes another image call image 4. Apply OR operation in between cipher image and image it makes the image 3. Using image 4 and image 3 apply XOR operation with key element image with respectively those makes image 1 and image 2 .

The followed figure will explained in detail of proposed method.
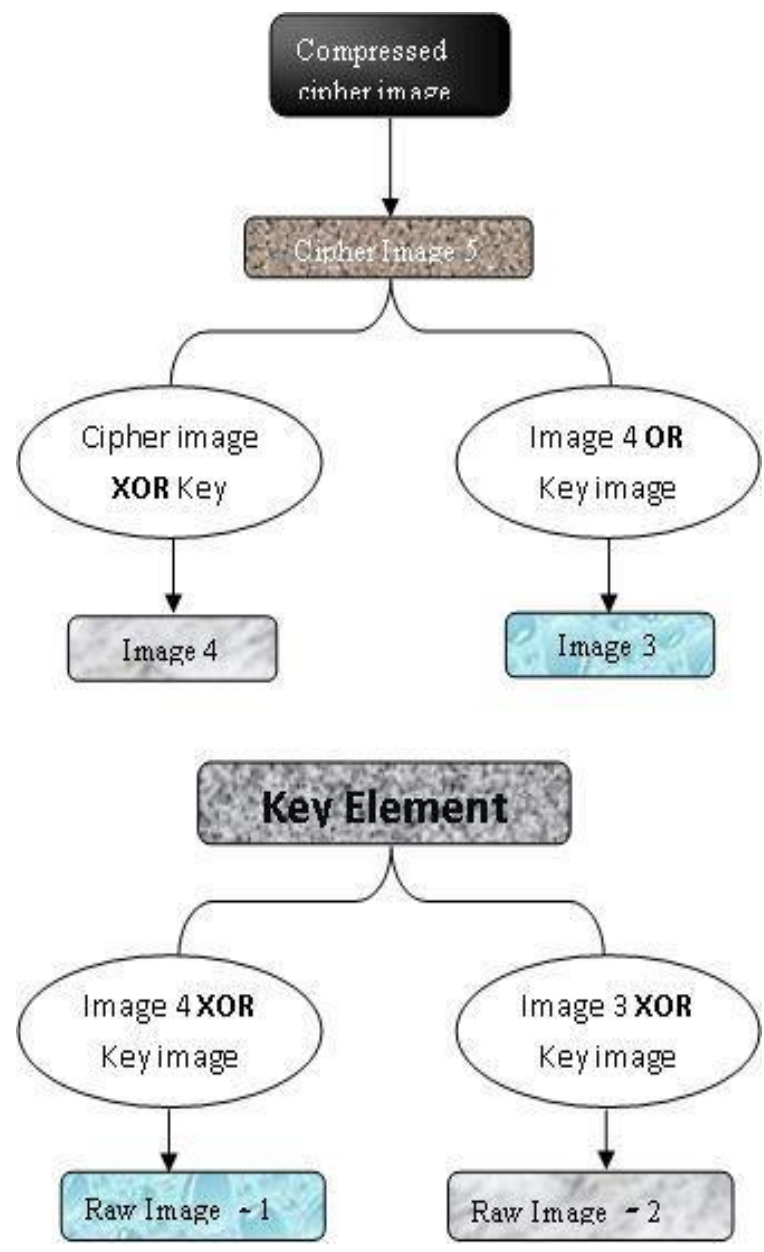

\section{Picture 2. Backward Technique for Proposed Method}

Step -1. Consider compresses cipher image 5 as an input files and read it.

Step - 2. Uncompress the input file, it makes only cipher image5.

Step - 3. Apply [(key element image) XOR (cipher image5)] == image 4

Step - 4. Apply [(cipher image 5) OR (cipher image4)] == image 3

Step - 5. Apply [(image -4) XOR (key element image)] = image -2 file.

Step - 6. Apply [(image-3) XOR (key element image)] = image -1 file.

\section{Results}

The followed table will shows the sizes of Raw, Encrypted and Compressed Images. 
Table 1. Size of Raw, Encrypted, Compression and Retrieved Images

\begin{tabular}{|c|c|c|c|c|c|c|}
\hline $\begin{array}{l}\text { S. } \\
\text { No }\end{array}$ & $\begin{array}{l}\text { Image } \\
\text { Name }\end{array}$ & $\begin{array}{l}\text { Raw } \\
\text { Image Size }\end{array}$ & $\begin{array}{l}1^{\text {st }} \text { Level } \\
\text { Key Image }\end{array}$ & $\begin{array}{l}2^{\text {nd }} \text { Level } \\
\text { Key Image }\end{array}$ & $\begin{array}{l}\text { Comp } 2^{\text {nd }} \text { Level } \\
\text { Key Img Size }\end{array}$ & $\begin{array}{l}\text { Retrieved } \\
\text { Image Size }\end{array}$ \\
\hline \multicolumn{7}{|c|}{$128 \times 128$ Image Sizes } \\
\hline \multirow[b]{2}{*}{1} & Pepper & 16384 & \multirow[t]{2}{*}{16384} & \multirow[t]{2}{*}{16384} & \multirow[t]{2}{*}{1453} & 16384 \\
\hline & Baboon & 16384 & & & & 16384 \\
\hline & \multicolumn{6}{|c|}{145 X 145 Image Sizes } \\
\hline \multirow[b]{2}{*}{2} & Lena & 21025 & \multirow[t]{2}{*}{21025} & \multirow[t]{2}{*}{21025} & \multirow[t]{2}{*}{1985} & 21025 \\
\hline & Women & 21025 & & & & 21025 \\
\hline & \multicolumn{6}{|c|}{255 X 255 Image Sizes } \\
\hline \multirow[b]{2}{*}{3} & OldMan & 65025 & \multirow[t]{2}{*}{65025} & \multirow[t]{2}{*}{65025} & \multirow[t]{2}{*}{49125} & 65025 \\
\hline & Family & 65025 & & & & 65025 \\
\hline \multicolumn{7}{|c|}{300 X 300 Image Sizes } \\
\hline \multirow[b]{2}{*}{4} & HotMan & 90000 & \multirow[t]{2}{*}{90000} & \multirow[t]{2}{*}{90000} & \multirow[t]{2}{*}{75325} & 90000 \\
\hline & Light Man & 90000 & & & & 90000 \\
\hline
\end{tabular}

When we observe the above table, the original image sizes are equals to different levels of key image sizes and retrieved image sizes. Than we applied a compression technique on $2^{\text {nd }}$ level encrypted images it will reduce the size of image and it is more helpful to transmits enven though low bandwidth communication also.

\section{Conclusion and Feature Work}

In this paper, Multi Medical Image Secure Hiding Technique at multi levels using Rational Operations and compression proposed. This algorithm is based on the standard of logical operations on image pixels. To perplex the relationship between original and encrypted images, using the OR, XOR operations are applied to two images and make different levels of encrypt images. Experimental tests have been carried out with detailed numerical analysis which demonstrates the robustness of the proposed algorithm against several types of attacks such as statistical and differential attacks (visual testing). Moreover, performance assessment tests demonstrate that the proposed image encryption algorithm is highly secure. It is also capable of fast encryption/decryption which is suitable for real-time Internet encryption and transmission applications.

\section{References}

[1] FengLiu State Key Lab. of Inf. Security, Chinese Acad. of Sci., Beijing, China, C. Wu and X. Lin, "Step Construction of Visual Cryptography Schemes", Information Forensics and Security, IEEE Transactions, vol. 5, no. 1.

[2] G. Thippanna, T. Bhaskara Reddy and S. Kiran, "Image Masking and Compression Using user Private Key Generation", IJETTCS ISSN 2278-6856, vol. 3, no. 5, (2014) September-October.

[3] http://blogs.epfl.ch/mmspgnews/documents/OP317Call.pdf.

[4] G. Jacob, "DNA based Cryptography: An Overview and Analysis", A. Murugan2 Int. J. Emerg. Sci., ISSN: 2222-4254@ IJES, vol. 3, no. 1, (2013) March, pp. 36-42.

[5] The Influence of Luminance on Local Tone Mapping Laurence Meylan and Sabine Süsstrunk, Ecole Polytechnique Fédérale de Lausanne (EPFL), Lausanne, Switzerland.

[6] G. Thippanna and T. Bhaskara Reddy, "A re-examine of gen on an assortment of images, compression techniques and its algorithms", International Journal of Advanced Research in Computer Science (IJARCS), http://www.ijarcs.info/?wicket:interface=:2:::: , 2012 November-December ISSN. 


\section{Authors}

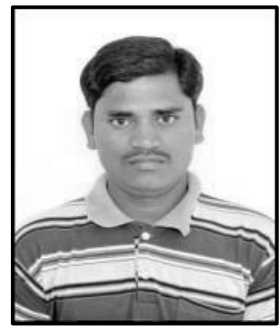

G. Thippanna, Research Scholar (Ph.D.). He has completed his Post Graduation in 2008 from S.V. University, Tirupati. He 4 years of teaching experience and 4 years of research experience, till now 4 international papers, 5 national conferences, and 3 national workshops. Email. gt.pana2012@gmail.com.

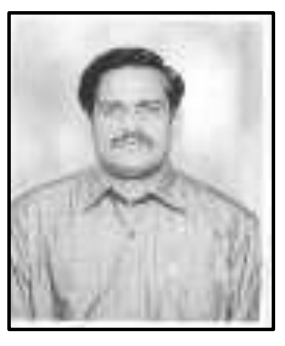

T. Bhsakara Reddy, Associate Professor. Department of Computer Science and Technology at S.K University, Anantapur A.P. He holds the post of Deputy Director of Distance education at S.K. University and He also the CSE Coordinator of Engineering at S.K. University. He has completed his $\mathrm{M}$. Sc and $\mathrm{Ph}$. D in computer science from S.K. University. He has acquired M. Tech from Nagarjuna University. He has been continuously imparting his knowledge to several students from the last 17 years. He has published 47 National and International publications. He has completed major research project (UGC). Four Ph. D and Three M. Phil have been awarded under his guidance. His research interests are in the field of image Processing, computer networks, data mining and data ware house. 